\title{
Eugénie Foa, sur les traces de Madame de Beaumont
}

Maria Eugenia Fernández Fraile

\section{(2) OpenEdition Journals}

Electronic version

URL: https://journals.openedition.org/dhfles/3071

DOI: $10.4000 /$ dhfles.3071

ISSN: 2221-4038

\section{Publisher}

Société Internationale pour l'Histoire du Français Langue Étrangère ou Seconde

\section{Printed version}

Date of publication: 1 June 1999

Number of pages: p.436-452

ISSN: 0992-7654

\section{Electronic reference}

Maria Eugenia Fernández Fraile, "Eugénie Foa, sur les traces de Madame de Beaumont", Documents pour l'histoire du français langue étrangère ou seconde [Online], 23 | 1999, Online since 26 August 2015, connection on 10 March 2023. URL: http://journals.openedition.org/dhfles/3071 ; DOI: https://doi.org/ 10.4000/dhfles.3071

This text was automatically generated on 10 March 2023.

All rights reserved 


\title{
Eugénie Foa, sur les traces de Madame de Beaumont
}

\author{
Maria Eugenia Fernández Fraile
}

1 Il y a des destins qui se rapprochent par des similitudes surprenantes, malgré la distance temporelle et spatiale. À lire les biographies de Madame Le-prince de Beaumont (1711-1780) et de Eugénie Foa, née Rodriguez Gradis (1798-1853), on est frappé par leurs ressemblances, dont certaines sont peut-être moins fortuites .qu'on ne le croirait, et d'autres sont certainement voulues, Eugénie Foa ayant cherché un modèle à suivre dans l'attitude de Mme de Beaumont, pour bâtir une vie indépendante, et par son engagement en faveur de l'amélioration du sort des femmes, au moyen de l'éducation des petites et des jeunes filles.

2 Toutes les deux proviennent de familles aisées (famille nobiliaire, dans le cas de Mme de Beaumont ; riche famille juive provenant d'Espagne, dans le cas d'Eugénie Foa, qui naît à Bordeaux, 1798)' ${ }^{1}$, ce qui leur donne accès l'acquisition de l'éducation élémentaire qu'on réservait aux jeunes filles à l'époque. Toutes deux sont mariées très jeunes; dans les deux cas, l'expérience conjugale tourne mal. Si c'est Mme de Beaumont qui abandonne son époux, il apparait que c'est Eugénie Foa qui est abandonnée par son époux (DBF). Dans les deux cas, toutefois, l'essentiel est - croyons-nous - que, au lieu de s'installer dans une vie maritale malheureuse, elles n'ont pas peur d'affronter une vie indépendante et difficile, l'une en prenant l'initiative d'une séparation, l'autre en ne se pliant pas aux exigences de son mari, en ne devenant pas la femme obéissante et silencieuse qu'on attendait qu'elle fût. ${ }^{2}$

3 Toutes deux sont réduites par leur séparation à une "situation financière des plus précaires » (DBF), et décident de gagner leur vie par elles-mêmes : Mme de Beaumont part en Hollande, puis à Londres, où elle est engagée comme préceptrice auprès d'une noble famille, puis plusieurs familles de la société londonienne lui confient l'éducation de leurs filles ; elle commence à écrire des articles pour un. journal ; elle devient journaliste, puis directrice d'un périodique, elle publie des essais, des lettres, des commentaires, des romans-feuilletons, se consacrant à partir de 1753 à publier des ouvrages pédagogiques destinés à l'éducation des jeunes filles, notamment le Magasin 
des Enfants (1756), dont on connaît la résonance dans toute l'Europe dans la seconde moitié du XVIII ${ }^{\mathrm{e}}$ siècle et pendant tout le $\mathrm{XIX}^{\mathrm{e}}$ siècle, et dont l'influence quant, à l'enseignement, du FLE a été mis en relief par Uta Janssens, et par $\mathrm{M}^{\mathrm{a}}$ Elena Romero Alfaro pour ce qui concerne l'Espagne, au Colloque d'Avila de 1997.

Quant à Eugénie Foa, « réduite au secours que lui accordait son père » (DBF $1976: 151$ ), elle décide d'écrire, de devenir écrivain. On ne peut, pas être d'accord avec les appréciations du $D B F$, qui distillent encore les préjugés masculins quand une femme décide d'exercer librement une profession : «Elle prit la plume, non par vocation, mais par nécessité. Écrire ne fut d'abord pour elle qu'un moyen d'existence, puis devint un amusement lorsqu'il s'avéra lucratif » (1976 : 151). Presque tous les écrivains, et pas seulement les femmes, ont écrit dans leurs débuts par nécessité (par exemple Balzac, à la même époque) ; quant à l'obtention du succès et de la reconnaissance des lecteurs, cela ne tombe pas du ciel, ce n'est pas le fruit du hasard comme le verbe "s'avérer » (= apparaître, se révéler ) donne à croire : c'est le fruit d'un long et sérieux travail, nullement un amusement, comme nous l'établirons par la suite.

Eugénie Foa se met donc à écrire : " elle composa et publia beaucoup de charmants ouvrages historiques et moraux pour les enfants et les jeunes filles " (DBF $1976: 152){ }^{3}$ Le choix de ce créneau du marché littéraire n'est nullement le fruit du hasard ni le fruit de la décision d'une fine et calculatrice marchande, qui a à l'œil ce qui est « lucratif» ou pas. La $B U$ nous signale l'élément clé de sa décision d'écrivain : elle " a donné divers romans et de nombreuses publications destinées à l'enseignement de la jeunesse » (BU 1966 : 267). Améliorer renseignement de la jeunesse, et notamment des jeunes filles, probablement pour que les générations futures ne connaissent pas les souffrances qu'elle a elle-même endurées : la littérature comme rachat d'une existence pénible, au moins dans les nombreuses années qui précèdent le succès, l'espoir d'être utile à d'autres, la volonté de changer un système social qui destine les hommes à la vie publique et les femmes au cloisonnement de la vie privée, la croyance en des valeurs qu'il faut transmettre, voilà la vocation d'Eugénie Foa. Pour peu que nous lisions ces « charmants ouvrages historiques et moraux ", on ne peut que se convaincre d'un choix délibéré d'Eugénie Foa, dès ses premiers ouvrages, dès ses premiers articles et contes publiés dans les journaux et gazettes qui commençaient à apparaître : Le journal des Enfants (1832-1897), La Gazette de la Jeunesse (1841-1845), Le Journal de l'Enfance (1842) ${ }^{4}$, Le Magasin des Enfants (1847-1848) ${ }^{5} .$.

6 La dédicace (à sa sœur Léonie, qui «n'a pas encore quitté le toit paternel ») des Six histoires de jeunes filles (1836) est révélatrice à cet égard : elle veut prouver « cette grande vérité : l'union fait le bonheur des familles ». Dans la Préface» elle montre clairement ses intentions, son choix :

Une chose dont on devrait le plus s'occuper, et que l'on néglige le plus souvent, c'est le choix des lectures des jeunes filles, depuis l'âge de treize ans jusqu'à leur mariage. - Il faut, disent quelques mères et quelques institutrices, que les demoiselles ne lisent que des choses sérieuses, instructives ; elles ont raison, moins le que. À tout âge, l'esprit a besoin de se récréer, et où voulez-vous qu'une jeune fille aille chercher ses lectures ? ce n'est, à coup sûr, ni dans les romans, ni dans les journaux, ni dans les revues.

II $y$ a cinq ans qu'un homme très remarquable pensa combler ce vide en fondant le Journal des Enfants (...) D'autres journaux sont encore spéciaux aux demoiselles. Mais ces publications sont encore loin de suffire à cette soif de lecture qui dévore le jeune âge. 
Tout en marchant sur les traces de M. Bouilly, dont les contes charmants ont récréé mon enfance, j'ai l'ambition d'y joindre une utilité de plus. Amuser est mon but, instruire en doit être le résultat ; pour cela, je crois qu'en composant quelques contes historiques, comme W. Scott créait ses romans historiques, en n'altérant nullement la vérité, en conservant religieusement les dates, en mettant mes personnages en action, et en les faisant parler et agir suivant leurs caractères connus, je contenterai les parents, les jeunes personnes, et moi. (1836: Préface).

Préface qu'elle signe : Eugénie Foa, née Rodriguès. L'adjonction de son nom de jeune fille ne peut pas être fortuite : elle marque la revendication de sa personnalité propre, séparément de celle de son mari. L écriture comme utilité publique (amusement, instruction), mais aussi comme fruit d'une satisfaction personnelle («je contenterai les parents, les jeunes personnes, et moi ») marque une volonté délibérée, une vocation, une conscience très nette de ce qu'elle veut faire dans sa vie.

Et dans ce choix, elle rencontre l'exemplede Mme Lepricede Beaumont. On nepeut pas établir à quel moment de sa vie elle connaît les œuvres de Mme Leprince. La Préface antérieure nousfait voir que pendantsa jeunesse elle n'apas eu l'occasionde lire ses œuvres. En effet, Eugénie Foa publie une réédition du Magasin des Enfants, par Mme Leprince de Beaumont (1846, Paris, Librairie Pittoresque de la Jeunesse, $2^{\mathrm{e}}$ éd.), oùelle «cherche à rajeunir » le livre de Mme Leprince de Beaumont, qui avait un peu vieilli : « Puis j'ai placé l'action en France, au lieu de la fairepasser en Angleterre.J'aichangé en noms français les noms anglais »(1846 : Préface).Celle réédition n'était nullement une opération commerciale, puisqu'elle connaissait déjà un énorme succès et vivait confortablement. C'est plutôt un hommage qu'elle rend à Mme Leprince, désirant que la jeunesse de son époque continue de lire son ouvrage.

9 Cet hommage, elle le lui rend aussi en plaçant un petit conte, "L'hôtel des trois couronnes ", en guise de préface. Je reproduis les principaux paragraphes de cette petite histoire pour en savourer tout son charme, tout en résumant certains éléments :

En 1748, au début de l'hiver, une damese présenteà l'hôtel des Trois Couronnes à Londres. Elle s'installe dans une chambre dont le loyer n'est pas cher, ne prend aucun deses repas à l'hôtel ; elle n'a pas d'argent: elle va acheter elle-même à la halle de quoi manger, elle n'a même pas les moyens d'avoir une chauffette pour se chauffer... Elle passe toute la journée à écrire, ne sort qu'un quart d'heure lematin. Pendant deux quinzaines, elle vît de la sorte. La deuxième quinzaine, « elle sortait plus souvent, écrivait moins par conséquent, et chaque fois qu'elle rentrait, elle paraissait plus accablée et découragée que fatiguée ; souvent ses yeux baignés de larmes se détournaient avec affectation de ceux que le hasard ou un sentiment de curiosité malveillante mettait sur son chemin.

Un jour, sur le même palier, un bruit se fit entendre : l'hôtesse, Mistress Green, est en train de chasser de l'hôtel une jeune fille qui ne peut plus payer son loyer. " Orpheline, sans appui, élevée dans une pension dont la maîtresse est morte il y a 2 mois ", elle espérait travailler comme institutrice. Mais on la trouve trop jeune partout Elle supplie Mistress Green de lui accorder un peu plus de répit. Au bruit des imprécations, la foule augmente. La dame française l'accueille ; elles rentrent toutes deux dans sa chambre.

Mistress Green laisse voir son dédain envers elle, elle n'a pas d'argent elle non plus ! Mais un des locataires l'a reconnue : « je la croyais riche »; «c'est une dame du plus haut mérite. L'innée dernière, j'étais à Commercy au moment où elle offrit au roi de Pologne in roman de sa composition, Le Triomphe de la Vérité ». Il dit à Mistress Green d'aller demander à Madame de Beaumont l'honneur d'une visite. Elle ne lui cache pas qu'elle désirait obtenir à Londres une place d'institutrice dans une grande maison. Celui-ci s'engage à la lui procurer. Elle est engagée chez Lady 
***, qui lui confie l'éducation de ses filles. La jeune fille trouve aussi une place de sous-maîtres se dans une pension, par les soins de Mme de Beaumont en plus de rapporter cette extraordinaire anecdote Eugénie Foalui rendhommage enprésentant unecourte biographie de Mme Leprince, destinée à faire revivresa mémoire, biographie digne d'une pierre tombale parsa simplicité et sa profondeur, où son admiration pour cette femme selaisse nettement transparaître :

Mme MarieLeprince née, àRouen le 26avril1711, épousa un M.de Beaumont qui ne la rendit pas heureuse, puis, après avoir consacré 17 années de sa vieàLondres, àl'éducation de jeune ladys, elle semaria en secondes noces à M. Thomas Pichon. Devenue mère de six enfants, elle sentit le besoin de seconsacrer à eux. Elle acheta en 1768, du fruit de ses économies, la petite terre de Chavanod, dans les environs d'Annecy, en Savoie, où elle se retira avec son mari et ses enfants. Elle y composa encore quelques ouvrages sur l'éducation, et y mourut, en 1780, à l'âge de 69 ans (1846).

11 Ce n'était nullement par simple amusement qu'Eugénie Foa écrivait. L'amplitude de sa production littéraire, que nous signalons dans les Annexes, atteste son labeur, son effort, son engagement, sa vocation littéraire en somme :

Les nombreux articles qu'elle donna au Journal des enfants, au Journal des demoiselles, au Dimanche des enfants contribuèrent à la faire connaître et à asseoir sa réputation. Elle s'essaya aussi dans le roman, et quelques journaux quotidiens ont inséré dans leurs feuilletons des nouvelles qu'elle signait Maria Fitz-Clarence. (DBF 1976 : 152).

Pourquoi l'emploi de ce pseudonyme dans ses romans-feuilletons ? Pour se démontrer qu'elle était capable de triompher comme écrivain indépendamment du prestige atteint par le nom d'Eugénie Foa? connaître une vieillesse calme et heureuse. Au contraire, " pendant les dernières années de sa vie, Mme Foa eut à supporter de cruelles souffrances. Affligée d'une cécité complète, elle mourut en avril de 1852 ou 1853 » (DBF 1976 : 152). ${ }^{6}$

Les œuvres d'Eugénie Foa ne sont pas destinées à l'enseignement/apprentissage du FLE, en principe. C'est le mouvement inverse, par rapport au Magasin des enfants, qui se produit : si dans le cas de Mme Leprince, celle-ci conçoit son œuvre pour enseigner la langue française, en principe, à de petites et de jeunes filles, et aussi de jeunes garçons, à travers des lectures adaptées à leur niveau linguistique et à leur âge ; et à partir de là, comme moyen vers l'acquisition d'une éducation générale, pour forger leur caractère (" on s'applique autant à leur former le cœur qu'à leur éclairer l'esprit »), ${ }^{7}$ et pour faire des jeunes filles « des logiciennes, des géomètres, des philosophes, leur apprendre à penser pour vivre juste $»{ }^{8}$

En ce qui concerne Eugénie Foa, ses ouvrages sont d'abord conçus comme livres de lecture pour dé jeunes filles et déjeunes garçons, en langue maternelle. Les éditeurs commencent à publier des collections d'ouvrages d'une même matière ou destinés à un certain public : des bibliothèques de la jeunesse voient le jour. Ainsi, de nombreuses rééditions des œuvres d'Eugénie Foa ajoutent des sous-titres révélateurs : " contes historiques dédiés à la jeunesse ", " A nos jeunes lectrices et lecteurs ", " modèles de jeunes filles "... De même, toute une série d'ouvrages porteront le (sous-)titre de robinsonades à partir du succès du Petit Robinson de Paris, publié en 1840. Ouvrages, mais aussi exemplaires de journaux et de gazettes qui ont été utilisés postérieurement, dans 
les différents pays de l'Europe ou de l'Amérique, comme moyen complémentaire d'apprentissage du FLE. Ainsi, on trouve plusieurs de ses œuvres à la Bibliothèque du Palacio Real de Madrid, que les jeunes fils ou filles des rois et de la haute noblesse espagnole qui fréquentait la cour ont sûrement lues : Contes historiques, Courage et résignation, Les petits musiciens, Petits princes et petites princesses : Contes historiques, Les petits savants, Six histoires de jeunes filles, Vertus et talents, Les vocations ou les élus des BeauxArts...

16 Aussi, certaines éditions ou rééditions de ses œuvres sont-elles adaptées pour servir de livre de lecture pour la langue française : les Contes biographiques (1865, New York, Holt) sont accompagnés d'un vocabulaire pour en faciliter la compréhension, de même que le Petit Robinson de Paris, ou le triomphe de l'industrie (1864, New York, Holt ; Boston, C. Schoenhof), ainsi que les rééditions postérieures (Boston, Urbino : 1866, 67, 68, 69, et 70). Ou encore, certaines œuvres sont traduites pour servir de lecture en langue anglaise, à l'intérieur d'une institution scolaire ; c'est le cas des contes biographiques ou Biographical Sketches, by the graduating class of St. Joseph's Academy (traduction du français par N.Y. O'Shea), publiés en 1877 à Boston, Flushing, L.L, réédition 1879.

Lectures complémentaires d'une éducation scolaire qui veulent, comme Eugénie Foa l'indique, instruire et amuser : «Amuser est mon but ; instruire en doit être le résultat " (1836 : Préface). C'est aussi le but déclaré de la plupart des journaux où elle est collaboratrice :

C'est à tous les petits enfants que nous adressons cette feuille de papier [...] Il y a ici tout ce qu'il faut pour vous amuser et vous instruire [...] Je vais vous expliquer de quelle manière je prétends vous instruire [...] Je commencerai une jolie histoire, une histoire véritable, et qui, à ce titre, ne pourra manquer d'exciter votre intérêt, ce sera celle d'un de ces enfants qui se sont fait remarquer, dans leur jeune âge, par leur ardeur pour l'étude, et qui, plus tard, en ont été récompensés en devenant hommes de mérite. (Journal de l'enfance, janvier, 1842, $\mathrm{n}^{\circ} 1$ ).

Le moyen d'instruire en amusant, c'est principalement à travers des histoires vraies et exemplaires :

Ne vous attendez pas à trouver toujours, dans notre journal, les contes délicieux de votre nourrice et de votre bonne, des histoires d'ogres et de revenants qui font peur ; les aventures de Peau d'Âne et du Petit Poucet... Au lieu de vous faire des contes, nous vous dirons de l'histoire ; ce sera tout aussi amusant, tout aussi terrible que vos contes de fées; en même temps ce sera plus utile et plus vrai [...] Il faut savoir l'histoire pour être un homme et pour apprendre à se conduire en homme. Il faut savoir le passé pour prévoir l'avenir et pour comprendre le présent [...] Nous serons pour vous, non pas une bonne nourrice qui fait des contes, mais des historiens amusants, simples, véridiques et qui vous diront ce qui est arrivé avant vous, et ce qui arrive en même temps que vous. (Journal des enfants, juillet $1832, \mathrm{n}^{\circ} 1$ ).

Ou encore :

Ce n'est pas un de ces recueils frivoles destinés à n'éveiller dans l'esprit de leurs lecteurs qu'un vain intérêt de curiosité [...] C'est une œuvre où l'utile et l'agréable sont habilement entremêlés, où la science a sa place à côté du conte et de la nouvelle, où la morale emprunte des formes attrayantes, où la leçon marche toujours de pair avec l'amusement. (Gazette des enfants et des jeunes personnes, décembre $\left.1837, \mathrm{n}^{\circ} 1\right)$.

Eugénie Foa a spécialement collaboré à des journaux et des gazettes destinées à des jeunes filles ou des femmes : ainsi, Le Magasin des demoiselles (1844-1895), La Mère institutrice l'institutrice mère (1834-1865), Le messager des demoiselles (absorbée par La 
Gazette de la Jeunesse). Le pari en faveur d'une éducation des femmes est spécifiquement posé :

Les femmes d'aujourd'hui aspirent à pouvoir suivre le progrès des sciences, de la littérature et des arts ; elles savent qu'au sein de notre société, il n'existe plus que deux classes de femmes : celles qui ont reçu une bonne éducation et celles qui, dans la famille et dans le monde, manquent de cet élément de bonheur [...] Nous voulons à la fois être un journal et une encyclopédie pleine de morale et d'instruction, qui restera dans toutes les bibliothèques. (Le Magasin des demoiselles, octobre $1844, \mathrm{n}^{\circ} 1$ ).

Le but que se fixe La mère institutrice et l'institutrice-mère est de " s'occuper de l'éducation des femmes, qui, par leur salutaire influence, peuvent seules diriger vers le bien la génération nouvelle » (D. Lévi, avril 1839).

Les œuvres et les articles d'Eugénie Foa comportent ainsi une contribution importante quant à la rénovation des idées pédagogiques de l'époque, mouvement auquel elle participe avec Pestalozzi ou d'autres pédagogues : la devise " amuser pour instruire » s'opposait nettement à d'autres devises de signe contraire : « la letra con sangre entra » étant la formulation la plus crue. Sa contribution fut non moins importante en faveur de l'éducation des femmes, par ses nombreuses prises de position, ses romansfeuilletons, ses histoires.

Eugénie Foa participe ainsi au vaste mouvement issu de l'esprit des Lumières du XVIII siècle et de la Révolution Française, qui croit dans les valeurs de l'éducation, qui estime que le progrès de l'humanité passe par l'instruction du peuple. Elle prodigue dans ses ouvrages des histoires qui vantent la valeur de la famille, le courage dans l'adversité, la bonté des personnes, la vertu récompensée, la confiance dans la raison et le mépris des pratiques chimériques et illusoires de la magie ou de la sorcellerie (comme le fait d'aller chez des tireuses de cartes pour connaître l'avenir), la valeur de la culture et de l'éducation par-dessus la beauté chez une femme... Valeurs morales traditionnelles, bourgeoises certainement, qui ne manquaient pas de constituer un pôle de progrès face à l'ignorance et l'irrationnel dans lesquels on laissait antérieurement les jeunes enfants et jeunes filles des couches populaires. Cette question mérite cependant une étude bien plus profonde que ces quelques réflexions esquissées ici.

\section{BIBLIOGRAPHY}

AMAT, dir. (1976), Dictionnaire de Biographie Française, Librairie Letouzey et Ané, Paris.

CNRS (1986), La presse d'éducation et d'enseignement, CNRS, Paris.

DIVERS (1971), The National Union Catalog Pre-1956 Imprints, Mansell, Londres.

DIVERS (1978), Enciclopedia Universal Ilustrada Europeo-Americana, Espasa Calpe, Madrid.

LEPRINCE DE BEAUMONT, M. (1846), Le Magasin des Enfants, Librairie Pittoresque de la Jeunesse, Paris, $2^{\mathrm{e}}$ éd. Contient le conte L'hôtel des trois couronnes et une notice sur l'auteur par Mme Eugénie Foa. JANSSENS, U. (sous presse), « Les Magasins (des Enfants, des Adolescents, etc.) de Marie Leprince de Beaumont dans l'enseignement privé et public du français en Angleterre, Hollande, Allemagne et 
autres pays d'Europe de la $2^{\mathrm{e}}$ moitié du XVII ${ }^{\mathrm{e}}$ siècle à la $2^{\mathrm{e}}$ moitié du XIX $\mathrm{X}^{\mathrm{e}}$ siècle », SIHFLES-

Colloque d'Avila.

MICHAUD, A. (1966), Biographie Universelle Ancienne et Moderne, Akademische Druck, Graz.

\section{APPENDIXES}

\section{Annexe I}

\section{Cuvres d'Eugénie Foa}

: Le Kidouschim. 4 vol.

: Philippe. 2 vol.

: La laide.

: Les Blancs et les Bleus. 4 vol. 18.33 : La Fiancée de l'exilé.

.1833 : Racket.

: La fermme à la mode.

: La Juive, histoire du temps de la Régence, Paris.

: Six histoires de jeunes filles, Paris, L. Janet. Contient les histoires suivantes : Mlle Cazotte ; La sorcière des trois Islets, ou Joséphine de la Pagerie ; Mlle de Lajolais, ou La courageuse enfant ; Les petits gâteaux, ou Sœur Marthe ; La paysanne de Thoraise ; Les orphelins de Saint Gra-tien, ou Fanchette Brulard ; Elisa Mercœur, ou la petite institutrice. 1838 : Contes à ma sœur Léonie.

1838 : Les petits marins.

: Contes historiques pour la jeunesse. 4 vol.

Rééd. : 1843, Paris-Leipzig, Desforges et Cie. Contient les contes suivants : T. I : Franklin ; Joseph Jacquard ; Jacques Callot ; Hortense et Eugène de Beauharnais ; Louise de Penthièvre, duchesse d'Orléans ; Jean Bart ; Marie de Beaurepaire. T. III : Le petit dessinateur ; Mme Louise de France ; Une conspiration d'échaudés ; Daniel Foé ; Que dix-huit sous; Le Robinson du Havre ; Le petit terrassier. T. IV : JJ. Rousseau ; Sainte Victoire ; Jacques Cook ; Les élèves d'Ecouen ; Antoine Galland ; W. Mozart ; Les deux sœurs d'Ecouen ; Marie Leczinska ; Marietta Tintorella.

Rééd. : Liège, Imp. H. Dessain, $4^{\mathrm{e}}$ éd. 1883 ?

: La dernière des Penthièvre. Paris.

: Les Mémoires d'un polichinelle. Paris.

Rééd. : 1840, Bruxelles, Société belge de Librairie, Hauman.

: Le petit Robinson de Paris, Paris, Ébrard.

Rééd. : 1845, in-16 ; s.a., Le petit Robinson de Paris, Boston, Urbino, et 1866, 1867,1868, 1869,1870 ; 1846, Philadelphia, H. Longstreet, et 1857 ; 18.. ? Paris, Magnin, Blanchard et Cie ; Paris, 186. ? ; 1864 ... avec vocabulaire, New York Holt-Boston, C. Schoenhof ; $19 .$. ?, Paris, L. Janet ; 1903, New York, American Book Co.

1840 : Contes historiques. 1840 : Le vieux Paris. 
: Mémoires d'une jeune fille devenue grande.

: Les contes de ma bonne. 1841 : Soirées du dimanche.

: Mémoires de Croquemitaine.

: Les Petits Peintres.

Rééd. : 18.. ?, Paris, Magnin-Blanchard et Cie. Contient les contes suivants : Buonarotti, le petit artiste ; Le corrège, ou le petit bûcheron ; Sébastien Gomèz, le mulâtre de Murillo ; David Tenniers, le joueur de cornemuse ; B.E. Murillo, le petit faiseur de bannières ; Antoine Watteau, le petit couvreur.

: Les Petits Musiciens.

Rééd. : 185. ?, Paris, L. Janet. Contient les contes suivants : Le petit chanteur de la Madone, ou J.B. Pierre Aloïs de Palestrina ; L'enfant de chœur, ou Michel Lambert ; Le petit pèlerin, ou Giuseppe Tartini ; Sepperl le timbalier, ou J. Haydn ; Le petit paysan saxon, ou Amédée Naumann ; Le petit sorcier, ou A.W. Mozart.

Rééd. : Paris, Bédelet, s.a. 1845 : Le Juvénile Keepsake.

: Alexandrine.

1849-50 : Bibliothèque historique de la jeunesse, Paris, 4 vol. (paru en feuilleton ?).

\section{Annexe 2}

Rééditions des œuvres de Eugénie Foa (ou attribuées à E. Foa) comportant des adaptations et des réaménagements du contenu.

S.a. : Vertus et talents. Modèles de jeunes filles, contes historiques, Paris-Strasbourg, Imp. Ad. Christophe, $3^{\mathrm{e}}$ éd.

S.a. : Suite aux contes historiques pour la jeunesse, Paris, Desforges.

: Petite Madame. Histoire du siècle de Louis XIV, New York, F. Gaillardet.

: Le livre de la jeunesse. Contes historiques, Paris, Bédelet. Biographies de : Saint Victoire ; Elie de Bourdeille ; Mme Leprince de Beaumont ; Mme de Genlis ; L'Abbé de l'Epée ; Berquin ; Saint Médard ; Valentin Du val.

Rééd. : 18.. ? : Nouveau livre de la jeunesse. Contes revus et corrigés, Paris, Bédelet.

: Nouveaux contes historiques, Paris, Librairie Pittoresque de la Jeunesse.

1855 : Vertus et talents, modèles des jeunes filles, Paris, Bédelet, $2^{\mathrm{e}}$ éd. Contient les contes suivants : Olga, grande duchesse de Russie ; Mlle de Marillac ; Mlle de Lussan ; Mme Cottin ; Mme Elisabeth ; Mme Campan ; Maire d'Orléans ; La reine Victoria.

1855 : Scènes et récits historiques, tirés de Vhistoire de France. Classés et revus par Elisabeth Muller, Paris, A. Bédelet. Contient les récits suivants : Vercingétorix ; Clotilde ; Sainte Geneviève ; Bertrade Batilde, ou la petite esclave ; Marguerite de Provence ; Un tournoi ; Louise de Lorraine ; Isabelle de France, ou la reine enfantine ; Mlle d'Aubigné, ou la petite créole Marie Leczinska, ou la conspiration; La seule couronne indestructible.

: Les petits artistes, peintres et musiciens, Paris, A. Bédelet. Contient les contes suivants : Guido ; Van Dyck ; Salvator Rosa ; J.B. Lulli ; Marietta Tintorella ; Callot ; Sébastien Gomez; Boieldieu. 
: Les petits guerriers, Paris, A. Bédelet. Contient les contes suivants : Turenne ; DuguayTrouin ; Kléber ; Eugène Beauharnais ; Jean Bart ; Hoche ; Napoléon I ${ }^{\mathrm{er}}$; Le général Foy.

?: Petits princes et petites princesses : contes historiques... par feu Mme Eugénie Foa, Paris, A. Bédelet. Rééd. : 1864 ?, 1871 ?, 1890 ? ; s.a., Paris, Typographie de Ad. Laine et J. Havard.

1858 : Les enfants illustres. Contes historiques, Paris, A. Bédelet. Contient les contes suivants : Jeanne d'Arc ; Guttenberg ; Gustave Wasa ; Sixte--Quint ; Rubens ; Gassendi, Catherine I. ; Mozart. 1866, $3^{\mathrm{e}}$ éd. 1858 : Les petits poètes et littérateurs, Paris, A. Bédelet. Contient les contes suivants : Le roi René ; Clément Marot ; Fénélon ; Bernardin de Saint Pierre ; Christine de Pisan ; Mme de Sévigné ; Daniel de Foe ; Mme de Staël.

18.. ? : Daniel Foe, Paris, Detached from her Les petits poètes, a story of Defoe's childhood. 1860 : Grandeur et adversité, enfance de femmes célèbres. Contes historiques dédiés à la jeunesse, revus et corrigés, Paris, A. Bédelet. Contient les histoires suivantes : Clotilde de France ; Bertrade ; Mme de Maintenon ; Mlle de Penthièvre ; Mlle de Bourk ; Pauline Tourzel ; La reine Hortense. 1860 : Travail et célébrité, Paris, A. Bédelet. Autre éd. : Travail et succès. Contient les contes suivants : Colbert ; Cornil Bath ; Sedaine ; J.J. Rousseau ; Galland ; Métastase ; Favart ; Le capitaine Cook. 1862 : Les vocations ou les élus des BeauxArts, contes historiques dédiés à la jeunesse, par feu Mme Eugénie Foa, Paris, A. Bédelet. Contient les contes suivants : Solario ; Claude Gelée, dit le Lorrain ; Adrien Brauer ; Sophie Chéron ; Bosio ; Soufflot ; Canova.

:The story of Cecil and his dog, or, The reward ofvirtue, New York, James Miller. Rééditions en $187 .$. ? et en 1885 .

: Courage et résignation, contes historiques dédiés à la jeunesse, Paris, Bédelet. Contient les contes suivants : Vercingétorix ; Grisel Raleigh ;

Mlle de la Fayette ; Oberlin ; Marie de Beaurepaire ; Deux princes d'Orléans ; Mme Campan. Rééd. : Paris, Imp. Simon Raçon et Cie, s.a. 1865 : Contes biographiques, avec vocabulaire, New York, Holt. Contient les contes suivants : Michel Ange, ou le petit artiste ; Sébastien Gomez, ou le mulâtre de Murillo ; Amédée Mozart, ou le petit sorcier ;J. Haydn, Sepperl le timbalier ; Antoine Watteau, le petit couvreur.

Rééd. : 1866, Boston, S.R. Urbino/New York, Leopold and Holt, 1867, 1868, 1876, 1886.

1865 : Les nouveaux Robinsons, aventures extraordinaires de deux enfants qui cherchent leur mère, Paris, Bédelet.

Rééd : 1893, Paris, Librairie d'Éducation.

Rééd. : 1928, The strange search, Philadelphia-Londres, J.B. Lippincott co, 1868 : Contes variés, histoire et fantaisie, Paris, Bédelet. Contient les contes suivants : Les enfants de la vallée d'Argélès ; Angélique Kaufmann ; Virginie Chesquière ; Suzanne Centlivre ; Jacques Lafitte.

: Boy artists, or sketches of the childhood, Boston, E.P. Dutton and Cie.

: Persévérance et renommée, contes historiques, Paris, Bédelet. 1869 : Héroïsme et candeur, Paris, Bédelet.

1877-1979: Biographical sketches, by the graduating class of Saint Joseph's academy. Trad. N.Y. O'Shea, Boston, Flushing, L.I. Contient les Vies de : M. Angelo Buonarrotti ; Antoine Watteau ; Sébastian Gomèz ; Joseph Haydn ; Mozart. 
1884 : The little Robinson of Paris, or, industry s triumph. A taie for youth, translated from $E$. Foa s book, New York, Burgess and Stringer. 1890 : Les petits savants : contes historiques dédiés à la jeunesse, par feu Mme Eugénie Foa, revus et corrigés, Paris, A. Bédelet, nouvelle édition.

Contient les contes suivants : Cujas ; Pothier ; Vaucanson ; Dupuytren ; Montesquieu ; Franklin ; Jacquard.

1895 : The boy life of Napoléon, afterwards empereor of the French, adapted and extended for American boys and girls, Boston, Lothrop Pub. Company.

1925 : Little R. Crusoe of Paris, told from the French of E. Foa,

Philadelphia-Londres, J.B. Lippicott.

1927 : Mystery of Castle Pierrefitte, New York, Longmans.

\section{Annexe 3}

Journaux et gazettes auxquels Eugénie Foa a collaboré ou qui ont publié des articles ou des contes d'Eugénie Foa.

Journal de l'Enfance (1842).

Le dimanche des enfants (1842-1851).

Gazette de la Jeunesse (1841-1845).

Gazette des enfants et des jeunes personnes (1837-1839).

Journal des enfants (1832-1897).

Journal des familles ( 1846-1847).

Livre de la Jeunesse : rédacteur unique (1843-1846).

Magasin des demoiselles (1844-1896).

Magasin des enfants (1847-1848).

La mère institutrice et l'institutrice mère (1834-65).

Le messager des demoiselles (1842).

La récréation (1879-83).

\section{NOTES}

1. II existe certains doutes quant à la date de sa naissance : la Biographie Universelle de Michaud (désormais BU) et l'Enciclopedia Universal îlusîrada (désormais EUI) donnent la date de 1798, date qu'on retrouve dans le prologue de The Story ofCecil and his dog (1865, New York, J. Miller) ; The National Union Catalog (désormais NUC) donne la date de 1795 (1971 : 340-343) ; le Dictionnaire de Biographie Française (désormais DBF) ne précise pas de date (« elle naquit à Bordeaux vers la fin du XVIII ${ }^{\mathrm{e}}$ siècle, on dit aussi en 1803 »).

2. On préfère cette explication à l'explication traditionnelle (si un homme abandonne une femme, c'est que celle-ci est insupportable) que le DBF laisse entendre, en affirmant que "son caractère [d'Eugénie Foa] semblait [...] dépourvu de toute sensibilité communicative ", contrairement à ses charmants ouvrages pour enfants 
3. Voir dans l'Annexe 1 la liste de ses ouvrages et dans l'Annexe 2 la liste des éditions comportant des adaptations et des réaménagements du contenu.

4. Voir l'Annexe 3 pour la liste des revues auxquelles elle a collaboré

5. Les chiffres entre parenthèses indiquent les années où les revues ont été publiées.

6. La date de 1853 est attestée dans l'EUIet dans NUC.

7. Voir Le Magasin des enfans, ou Dialogues entre une sage gouvernante et plusieurs de ses élèves de la première distinction, 1776, Dublin, Chez Cales Jenkin, page de couverture.

8. Voir Uta Janssens, références bibliographiques.

\section{AUTHOR}

\section{MARIA EUGENIA FERNÁNDEZ FRAILE}

Université de Grenade 